\title{
Wavelet analysis of the LF radio signals collected by the European VLF/LF network from July 2009 to April 2011
}

\author{
Flavia Righetti ${ }^{1{ }^{\star}}$, Pier Francesco Biagi ${ }^{1}$, Tommaso Maggipinto ${ }^{1}$, Luigi Schiavulli ${ }^{1}$, Teresa Ligonzo ${ }^{1}$, \\ Anita Ermini ${ }^{2}$, Iren A. Moldovan ${ }^{3}$, Adrian S. Moldovan ${ }^{4}$, Aydin Buyuksarac ${ }^{5}$, Hugo G. Silva ${ }^{6}$, \\ Mourad Bezzeghoud ${ }^{6}$, Michael E. Contadakis ${ }^{7}$, Dimitrios N. Arabelos ${ }^{7}$, Thomas D. Xenos ${ }^{7}$
}

\author{
${ }^{1}$ Università di Bari, Dipartimento di Fisica, Bari, Italy \\ ${ }^{2}$ Università di Roma "Tor Vergata", Dipartimento di Ingegneria Meccanica, Rome, Italy \\ ${ }^{3}$ National Institute of Earth Physics, Seismological Depth., Bucharest-Magurele, Romania \\ ${ }^{4}$ AZEL-Designing Group S.R.L., Bucharest-Magurele, Romania \\ ${ }^{5}$ Canakkale Onsekiz Mart University, Department of Geophysics, Canakkale, Turkey \\ ${ }^{6}$ Universidade de Évora, Escola de Ciências e Tecnologia, Centro de Geofisica de Évora e Departamento de Física, Évora, Portugal \\ ${ }^{7}$ Aristotle University of Thessaloniki, Department of Geodesy and Surveying, Thessaloniki, Greece
}

\author{
Article history \\ Received June 16, 2011; accepted September 22, 2011. \\ Subject classification: \\ Seismic risk, Intruments and techniques, Data analysis, Radio Network, Wavelet precursors.
}

\section{ABSTRACT}

In 2008, a radio receiver that works in very low frequency (VLF; 20-60 kHz) and LF (150-300 kHz) bands was developed by an Italian factory. The receiver can monitor 10 frequencies distributed in these bands, with the measurement for each of them of the electric field intensity. Since 2009, to date, six of these radio receivers have been installed throughout Europe to establish a 'European VLF/LF Network'. At present, two of these are into operation in Italy, and the remaining four are located in Greece, Turkey, Portugal and Romania. For the present study, the LF radio data collected over about two years were analysed. At first, the day-time data and the night-time data were separated for each radio signal. Taking into account that the LF signals are characterized by ground-wave and sky-wave propagation modes, the day-time data are related to the ground wave and the night-time data to the sky wave. In this framework, the effects of solar activity and storm activity were defined in the different trends. Then, the earthquakes with $M \geq 5.0$ that occurred over the same period were selected, as those located in a 300-km radius around each receiver/transmitter and within the 5 th Fresnel zone related to each transmitter-receiver path. Where possible, the wavelet analysis was applied on the time series of the radio signal intensity, and some anomalies related to previous earthquakes were revealed. Except for some doubt in one case, success appears to have been obtained in all of the cases related to the $300 \mathrm{~km}$ circles in for the ground waves and the sky waves. For the Fresnel cases, success in two cases and one failure were seen in analysing the sky waves. The failure occurred in August/September, and might be related to the disturbed conditions of the ionosphere in summer.

\section{Introduction}

Since 1980, studies of the interactions between seismic activity and disturbances in radio broadcasts have been carried out. Pre-seismic disturbances in very low frequency (VLF) radio signals that lie in the $20-60 \mathrm{kHz}$ frequency band have been investigated mainly in Japanese and Russian studies [Hayakawa and Sato 1994, Morgounov et al. 1994, Hayakawa et al. 1996, 2006, Molchanov and Hayakawa 1998]. At the same time, pre-seismic disturbances on LF (150-300 $\mathrm{kHz}$ ) radio broadcasts have been proposed mainly in Italian studies [Biagi et al. 2001a,b, 2005, 2006a, Biagi and Hayakawa 2002]. Generally, the radio data have been collected by receivers located on the ground. Recently, some possible seismic disturbances were revealed by the VLF radio signals recorded by the French DEMETER satellite, as presented by Molchanov et al. [2006] and Rozhnoi et al. [2007].

All of the previous disturbances are related to variations of some parameters in the ground, the atmosphere and the ionosphere. The ground variations, such as uplift and tilt, gas emissions, underground water level fluctuations, changes in groundwater chemistry, and changes in electrical resistivity of rocks, are clearly related to microfracturing processes that can occur during the preparatory phases of earthquakes [Rikitake 1975, 1987, King, 1984/85, Roeloffs 1988, Wyss and Dmowska 1997]. On the other hand, to justify the atmospheric (mainly ionospheric) disturbances, two different models have been proposed. The first model assumes direct 


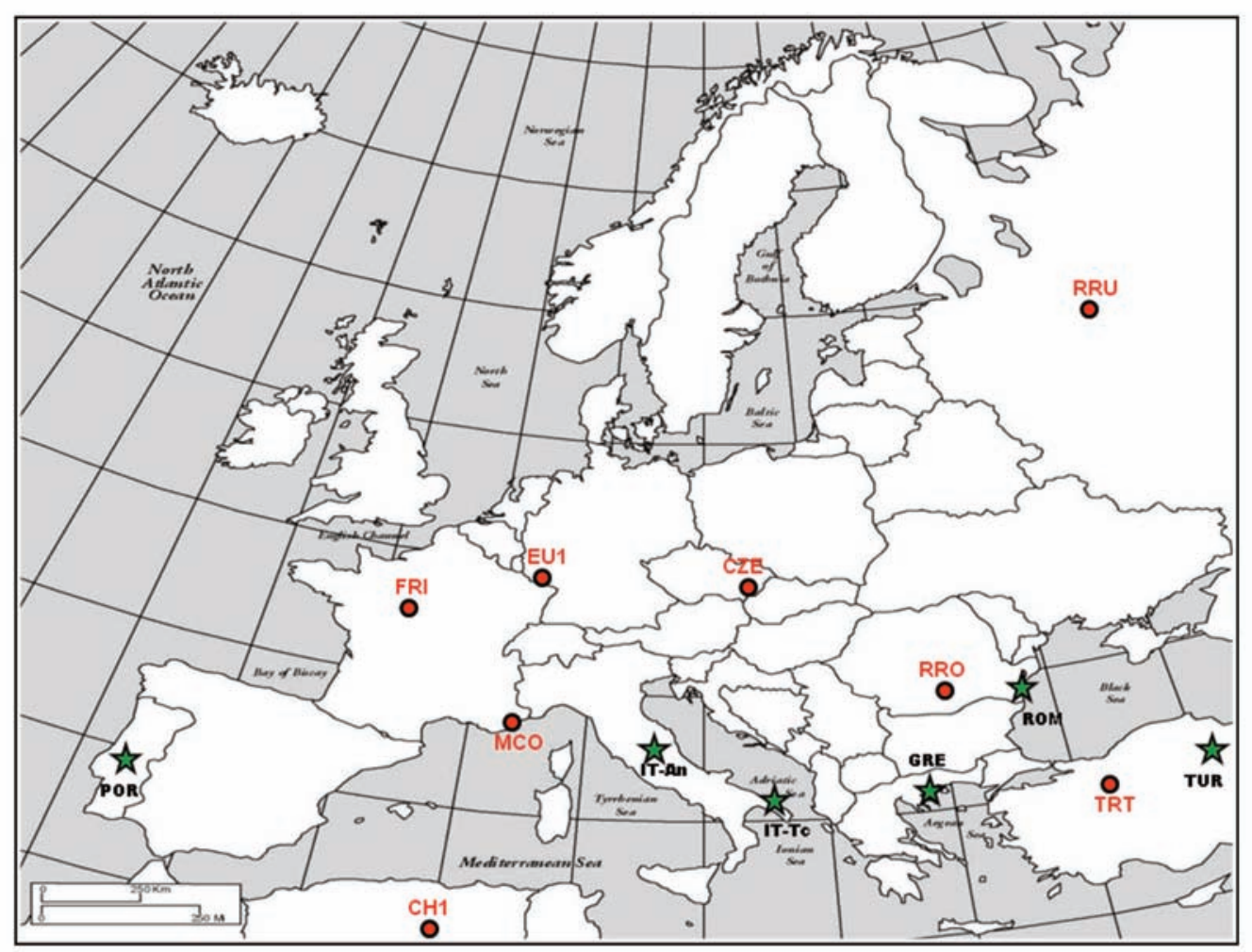

Figure 1. Map showing the locations of the receivers and the LF transmitters of the European Radio Network. Stars, receivers; circles, LF transmitters, the signals of which were collected by the different receivers. Some particularities of these transmitters are reported in Table 1.

\begin{tabular}{cccc}
\hline Code & Country & $\begin{array}{c}\text { Power } \\
(\mathrm{kW})\end{array}$ & $\begin{array}{c}\text { Frequency } \\
(\mathrm{kHz})\end{array}$ \\
\hline RRO & Romania & 1200 & 153 \\
FRI & France & 2000 & 162 \\
TRT & Turkey & 1200 & 180 \\
EU1 & Germany & 2000 & 183 \\
CH1 & Algeria & 2000 & 198 \\
MCO & France & 1200 & 216 \\
RRU & Russia & 2500 & 261 \\
CZE & Czech Republic & 500 & 270 \\
\hline
\end{tabular}

Table 1. Particularities of the LF transmitters of the European network.

effects of ionising radiation from gases (mainly radon) or aerosols, or electromagnetic emissions from the ground [Hayakawa and Sato 1994, Alperovitch 1997, Pulinets et al. 1998, Biagi et al. 2001b]; the second model assumes indirect effects of the production of gravity waves in the atmosphere/ionosphere [Hayakawa et al. 1996, Molchanov and Hayakawa 1998] as a consequence of pre-seismic processes in the ground. This second model overcomes the problem present in the first model, of the transport up to the ionosphere of particles or electromagnetic waves from the ground.

In any case, these results relating to the earthquake precursors obtained through the analysis of radio signals have been very encouraging, and these investigations are now becoming more widespread. In this framework, during
2008, a new radio receiver operating in both the VLF and the LF bands that can monitor the electric field intensities of 10 frequencies was developed by an Italian factory (Elettronika, Palo del Colle, Bari). Since 2009, to date, six of these receivers have been put into operation, two in Italy and the remaining four in Greece, Turkey, Portugal and Romania, giving rise to the first 'European VLF/LF Network'. A sampling rate of 1 min is used, and the electric field intensity of the signals is expressed in $\mathrm{dBm}$, as $\mathrm{dBm}=20 \log (\mathrm{Vm} \mathrm{Vpp})$. For simplicity, in the different Figures of this study that show data collected with these receivers, the $\mathrm{dBm}$ indication on the $\mathrm{y}$-axis is not reported.

The receivers and the network were described in detail by Biagi et al. [2011]. Figure 1 illustrates this radio network, and some particularities of the LF transmitters are listed in Table 1. We present here our first study of the LF data that was collected from July 2009 to April 2011.

\section{Theoretical features of LF radio signals}

LF radio signals are characterised by ground-wave and sky-wave propagation modes. The ground waves provide relatively stable signal. In contrast, the sky waves vary greatly from day to night, and in the day-time, from winter to summer.

For the ground waves, Rotheram [1981a,b] developed a model based on propagation over a smooth, homogeneous, curved earth with an exponentially decreasing refractive index. The meaning of an exponential atmosphere is that it 
represents the average atmospheric conditions more closely than a linear refractive index variation does. The factors that influence the propagation are: a) the refractive index $n$ of the troposphere; $b$ ) the scale height $h$ of the troposphere; and c) the permittivity $\varepsilon$ and the conductivity $\sigma$ of the ground. Here, $n$ and $\sigma$ are the most influential factors. Practically, the ground-wave propagation is affected by the troposphere and the ground conditions.

For the sky wave, the wave hop theory proposed by Knight [1973] can be considered. According to this theory, the sky-wave signals received by an antenna can be considered as rays that started from the transmitter and were reflected one or more times (hops) by the lower ionosphere and by the ground. For distances less than $1,000 \mathrm{~km}$, the sky wave is entirely represented by a one hop wave. The main factors influencing the propagation are: a) the ionosphere reflection coefficient $R$; b) the ionosphere focusing factor $D$; and c) the sky-wave path length $L$, which depend on the ionosphere reflection height. Practically, sky-wave propagation is affected by the ionosphere conditions mainly in the central part of the path.

\section{Data analysis}

\subsection{Night-time and day-time data}

As a first step, we separated the day-time data from the night-time data. To obtain datasets that are always related to the day-time and to the night-time regardless of the season, we selected the range from 08.00 to 13.00 (UT) for the daytime, and the range from 20.00 to 22.00 (UT) for the night-time. This night-time choice is also forced by the occurrence of an interruption of 3-4 $\mathrm{h}$ in some of the radio broadcasts, generally after the local 24.00. As an example, in Figure 2, the raw MCO transmitter signals collected during May 2010 by the IT-An receiver with the relevant night-time and day-time content are reported. On the basis of the results presented by Biagi et al. [2006b], we can consider that the night-time data represent the sky wave, while the day-time data represent the ground wave. Thus, according to the considerations made in the previous section, the sky wave might be affected mainly by the ionosphere conditions, and

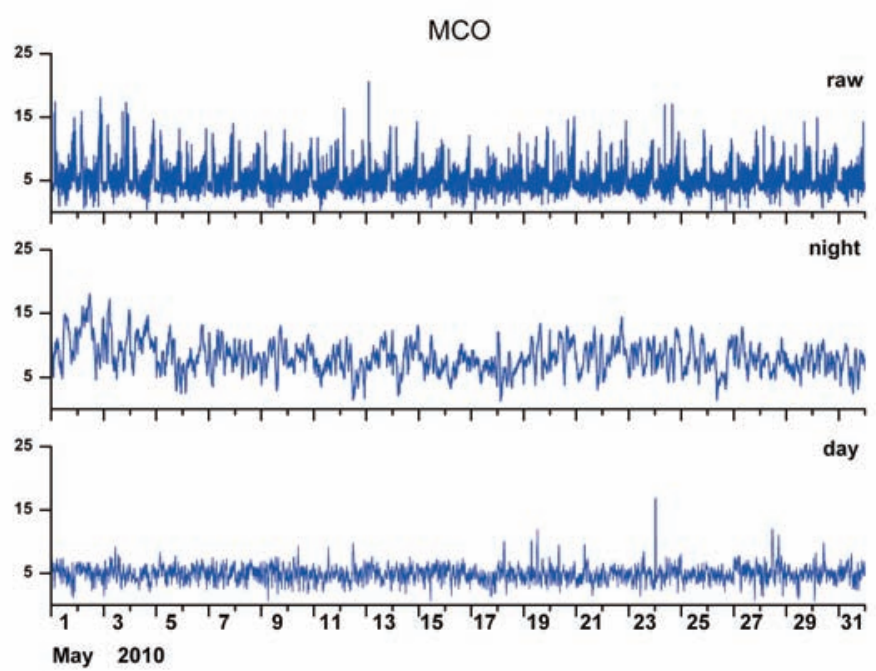

Figure 2. Night-time and day-time MCO transmitter radio signals collected by the IT-An receiver in May 2010. Top panel: Intensity of the raw MCO $(216 \mathrm{kHz})$ transmitter signals. Middle panel: Night-time $(20.00-$ 22.00 UT) data extracted from the raw data. Bottom panel: Day-time (08.00-13.00 UT) data extracted from the raw data.
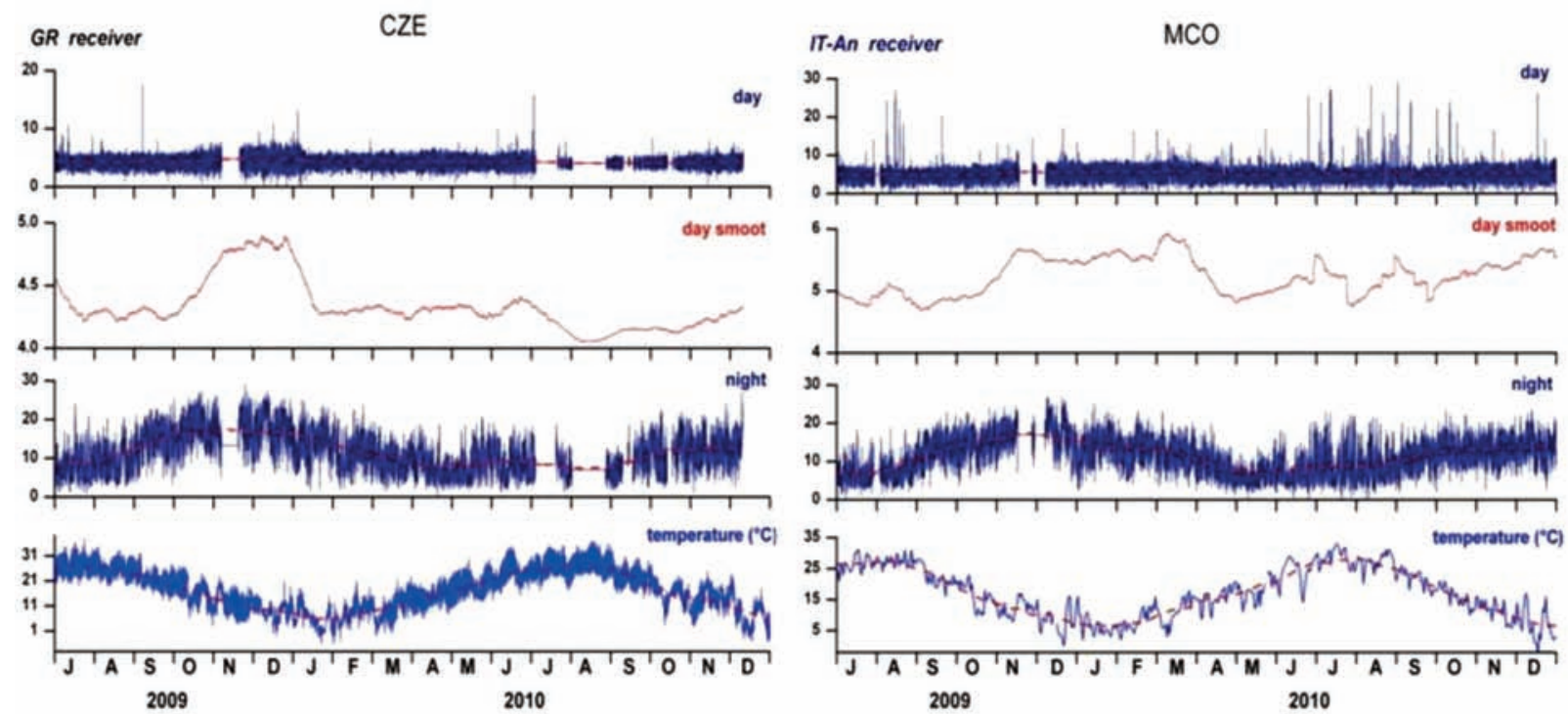

Figure 3. Day-time and night-time transmitter radio signals and local air temperature trends collected from July 2009 to December 2010 . Left: Intensities of the CZE $(270 \mathrm{kHz})$ transmitter signals collected by the GR receiver (Figure 1). Right: Intensities of the MCO (216 kHz) transmitter signals collected by the IT-An receiver (Figure 1). The local air temperature trends are also reported for both. Red dashed lines, long-term smoothed data. The second panels from the top show the 30 -day adjacent averaging smoothing of the day-time radio data. 
the ground wave by the troposphere/ground conditions.

To check these influences, we examined the night-time and day-time radio data collected by the different receivers over about two years, together with the data sampled by meteorological stations located in each receiver area. Figure 3 shows the night-time and day-time CZE transmitter radio signals collected by the GR receiver, and those of the MCO transmitter radio signals collected by the IT-An receiver, together with the air temperature trends in the two receiver areas over the same period.

A very clear correlation appears between the night-time radio signals and long-term temperature trends, demonstrating the influence of solar radiation on the ionosphere, and consequently on the night-time radio signals that represent the sky wave. As the solar radiation disturbs the ionosphere and consequently the reflection of the sky wave, the correlation ratio between these variations is negative. A lag between the temperature and radio-signal variations can be clearly seen in Figure 3, which indicates the delay of the heating/cooling on the ground with respect to that in the ionosphere, as would be expected. In Figure 3, the long-term trend of the day-time radio (dashed red lines in the plots) appears to be relatively stable, as must be expected for a ground wave. There are only some weak variations that can be seen in the radio data (Figure 3, second panel from the top), showing that there is some influence of the ground/troposphere conditions on the radio signal propagation.

Finally, Figure 4 shows the TRT transmitter day-time radio signals collected by the GR receiver, and the MCO transmitter day-time radio signals collected by the ROM receiver, together with the storms detected in the areas of the two receivers over the same period. The disturbances in the radio signals caused by the electricity produced by lightning during the storms are evident. This effect stands out clearly on the day-time radio signals data because the lightning activity occurs mainly during the day, and also because of the lower level of the day-time signal with respect to the night-time signal.

\subsection{Earthquake selection}

To uncover possible seismic effects on the radio signals, at first it was necessary to select the relevant earthquakes. The European Mediterranean Seismological Centre (EMSC) bulletin from July 2009 to April 2011 was used (EMSC website, http://www.emsc-csem.org). The three following criteria were adopted for the choice of the earthquakes: (a) earthquakes with $M \geq 5.0$ located inside the 5th Fresnel zone of the different radio paths; (b) earthquakes with $M \geq 5.0$ that occurred inside a circle with a $300-\mathrm{km}$ radius around each receiver; and (c) earthquakes with $M \geq 5.0$ that occurred inside a circle with $300-\mathrm{km}$ radius around each transmitter. Requirement (a) takes into account several results that have indicated that the area inside the 5th Fresnel zone is the most sensitive for seismic disturbances on radio propagation [Molchanov and Hayakawa 1998, Molchanov et al. 2006, Rozhnoi et al. 2004]. Requirements (b) and (c) are based on the dimensions of the area affected by possible pre-seismic effects [Dobrovolsky et al. 1979, Kingsley et al. 2001]. The results of these selections are reported in Table 2 . The cases denoted with an asterisk in Table 2 cannot be investigated due to a lack of radio data or for known local disturbances on the receiver [Biagi et al. 2011].
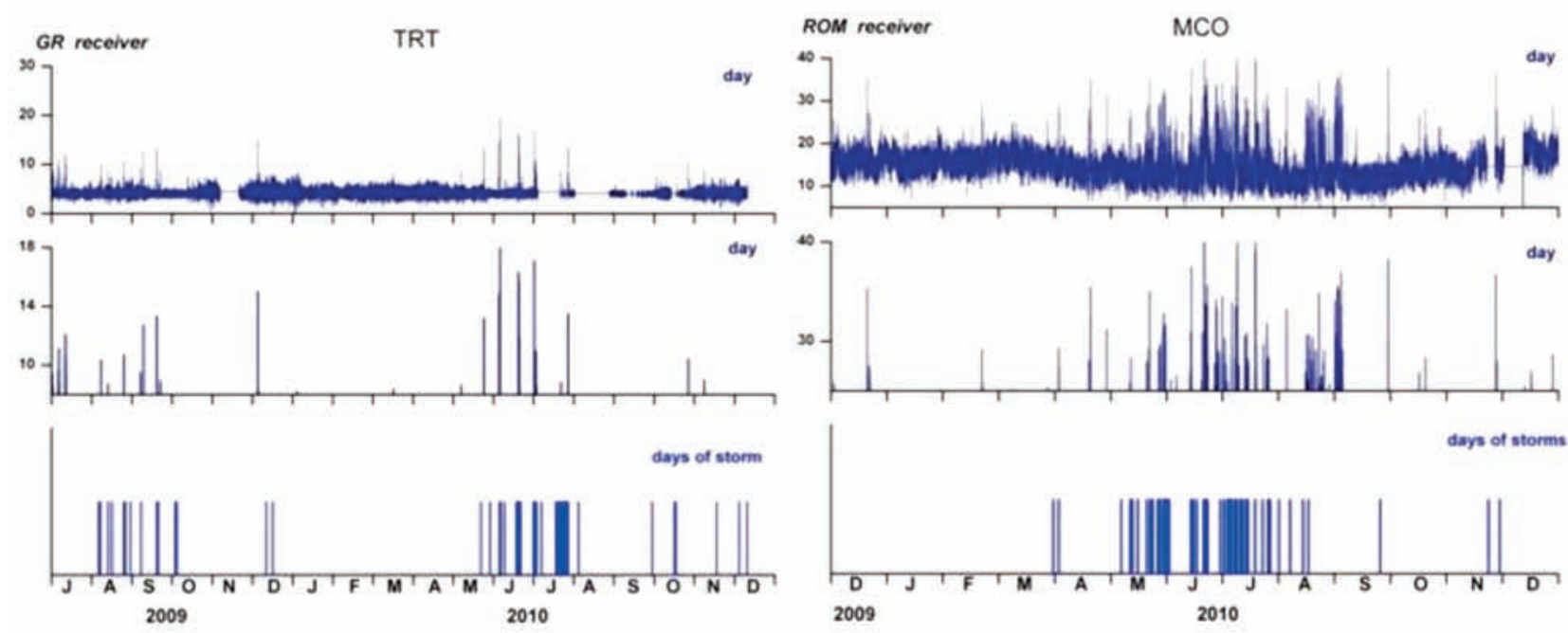

Figure 4. Day-time transmitter radio signals and storm days collected from July 2009 to December 2010. Left: Intensities of the TRT (180 kHz) transmitter signals collected by the GR receiver (Figure 1). Right: Intensities of the MCO (216 kHz) transmitter signals collected by the ROM receiver (Figure 1). The storm days are also reported for both. The second panels from the top show the day-time intensities on an enlarged scale to clearly show correspondence with the storms. 


\begin{tabular}{cccc}
\hline Radio path & \multicolumn{3}{c}{ Relevant earthquakes selected } \\
\cline { 2 - 4 } & $\begin{array}{c}\text { Coordinates } \\
\left({ }^{\circ} \mathrm{N},{ }^{\circ} \mathrm{E}\right)\end{array}$ & $\begin{array}{c}\text { Date } \\
\text { (yyyy-mm-dd })\end{array}$ & Magnitude \\
\hline Fresnel zones & & $2009-08-21$ & 5.0 \\
GR/MCO & $41.86,19.16$ & 5.6 \\
GR/MCO & $41.46,20.41$ & $2009-09-06$ & 5.3 \\
GR/TRT & $40.43,26.30$ & $2010-11-03$ & 5.4 \\
GR/CZE & $43.74,20.69$ & $2010-11-03$ & 5.0 \\
IT-Tc/RRO $\left(^{\star}\right)$ & $41.86,19.16$ & $2009-08-21$ & 5.0 \\
ROM/CH1 $\left(^{\star}\right)$ & $41.86,19.16$ & $2009-08-21$ & 5.6 \\
\hline Receiver circles & & & 6.0 \\
IT-Tc & $41.46,20.41$ & $2009-09-06$ & \\
TUR & $38.84,40.00$ & $2010-03-08$ & 5.0 \\
\hline Transmitter circles & & & \\
CZE & $51.45,16.11$ & $2010-02-06$ & \\
\hline
\end{tabular}

Table 2. Earthquakes selected according to the requirements indicated in Section 4.

\section{Wavelet analysis}

To reveal any anomalies on the radio data, the wavelet transform [Torrence and Compo 1998] was applied. In this way, it was possible to highlight the spectral components of the signals using variable-width time windows, by considering that the frequency content of these windows has an inverse relationship to the time widths. Thus, the localization of the signals is obtained simultaneously in both time and frequency [Daubechies 1992, Strang and Nguye 1996]. In our analysis, we adopted the 'Morlet function' as the wavelet [Torrence and Compo 1998]. In this case the wavelet transform of a time signal is a complex series that can be usefully represented by its square amplitude, i.e. we consider the so-called wavelet power spectrum. The power spectrum is a two-dimensional plot that once correctly normalized with respect to the power of the white noise, gives information on the strength and precise time of occurrence of the various Fourier components that are present in the original time series. Furthermore, the wavelet power corresponding to the Fourier period in which the maximum power is found can be usefully reported; i.e. the
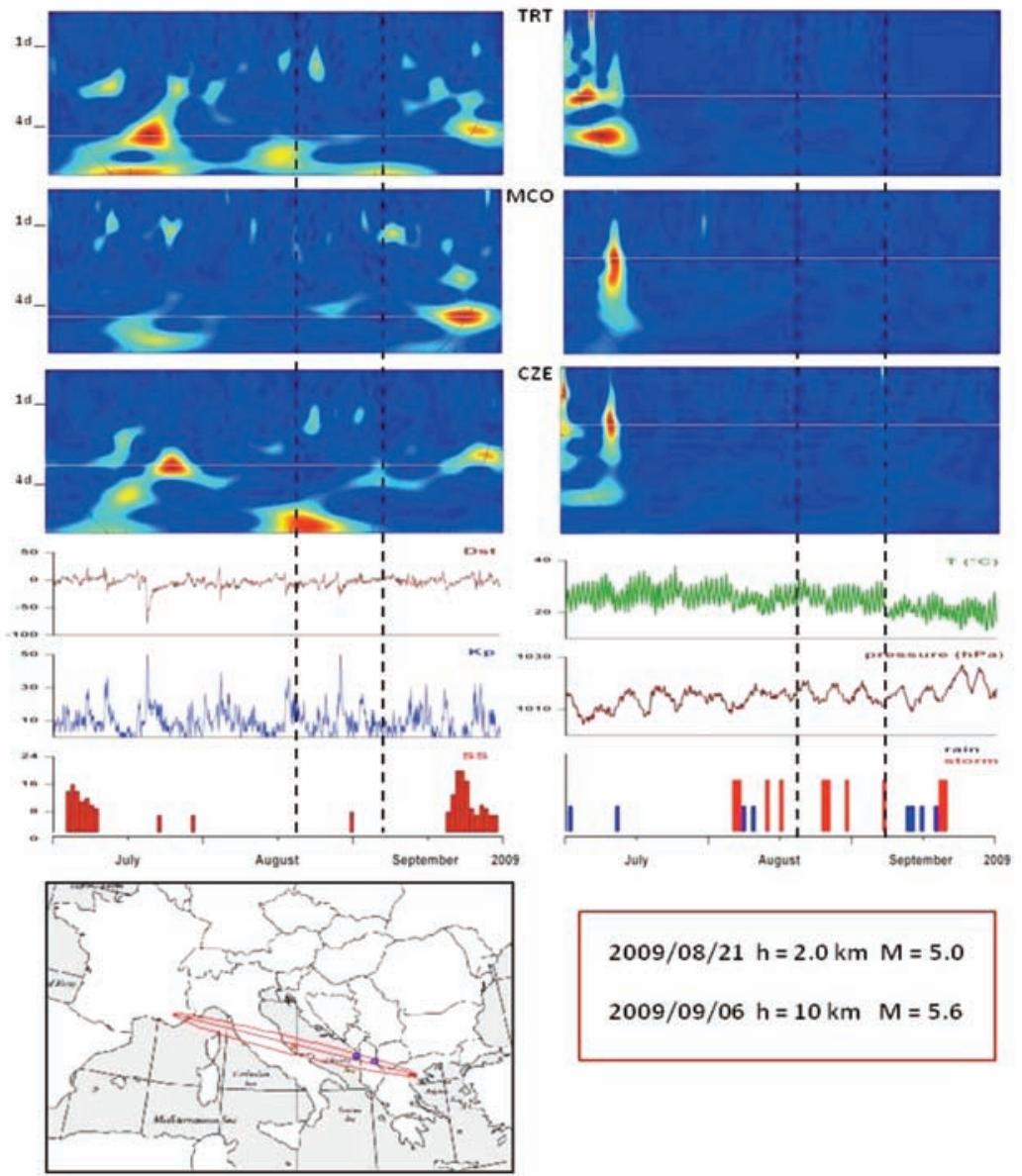

$2009 / 08 / 21 \mathrm{~h}=2.0 \mathrm{~km} \mathrm{M}=5.0$

$2009 / 09 / 06 \mathrm{~h}=10 \mathrm{~km} \mathrm{M}=5.6$

Figure 5. Night-time and day-time spectrograms of the TRT $(180 \mathrm{kHz}), \mathrm{MCO}(216 \mathrm{kHz})$ and CZE $(270 \mathrm{kHz})$ transmitter radio signals collected by the GR receiver, together with the geomagnetic/solar and the (local) meteorological data, from July to September 2009. Top panels: Spectrograms of the nighttime (left) and day-time (right) radio signals. Continuous black lines, delimitation of the region (COI) of the wavelet spectra in which edge effects become important (Torrence and Compo, 1998). Centre panels: Dst and Kp geomagnetic indices and solar burst numbers (SS) (left), and air temperature, air pressure and occurrence of rain and storms (right). Bottom: map showing the 5th Fresnel zone of the GR/MCO path, showing the epicentres of the August 21 and September 6, 2009, earthquakes that occurred inside these zones. Vertical dashed lines, time of occurrence of these earthquakes. 
one-dimensional plot that represents a single period with respect to time.

For the earthquakes that occurred in the Fourier zones, we looked for anomalies in the night-time data (the sky wave); the day-time data (the ground wave) were examined only to identify any anomalies in the night-time data related to some local day/night cause or to meteorological disturbances (in addition to the meteorological data). For the cases within the $300 \mathrm{~km}$ circles, both the night-time and daytime data were examined; indeed, in these cases, the seismicity produced local effects and so disturbances in both of these signal should to be detectable.

The results of this analysis according to the cases indicated in Table 2 are reported in Sections 4.1, 4.2 and 4.3.

\subsection{The Fresnel zones}

Figure 5 shows the analysis of the first two cases reported in Table 2 .

Figure 5 reports the night-time and day-time spectrograms of the TRT, MCO and CZE transmitter radio signals collected by the GR receiver, together with geomagnetic/solar and (local) meteorological data. The times of occurrence of the two earthquakes that occurred inside the 5th Fresnel zone of the GR/MCO path are indicated. These earthquakes occurred close together and not very far apart from each other in time, so a unique perturbation can be expected. As can be seen in Figure 5, a clear anomaly appears simultaneously in the different day-time spectrograms at the beginning of July. In our opinion, this anomaly is related to some local diurnal phenomenology that occurred in the receiver zone. For the night-time spectrograms, some anomalies appeared, although no particular correspondence with these earthquakes stands out. At the same time, the geomagnetic/solar conditions also cannot justify the anomalies. Therefore, irregular disturbances in the ionosphere that are typical of the summer can in all probability be claimed to justify these anomalies.

Figure 6 shows the analysis of the next two cases in Table 2, with the night-time and day-time spectrograms of the TRT, CH1 and CZE transmitter radio signals collected by the GR receiver reported together with the geomagnetic/
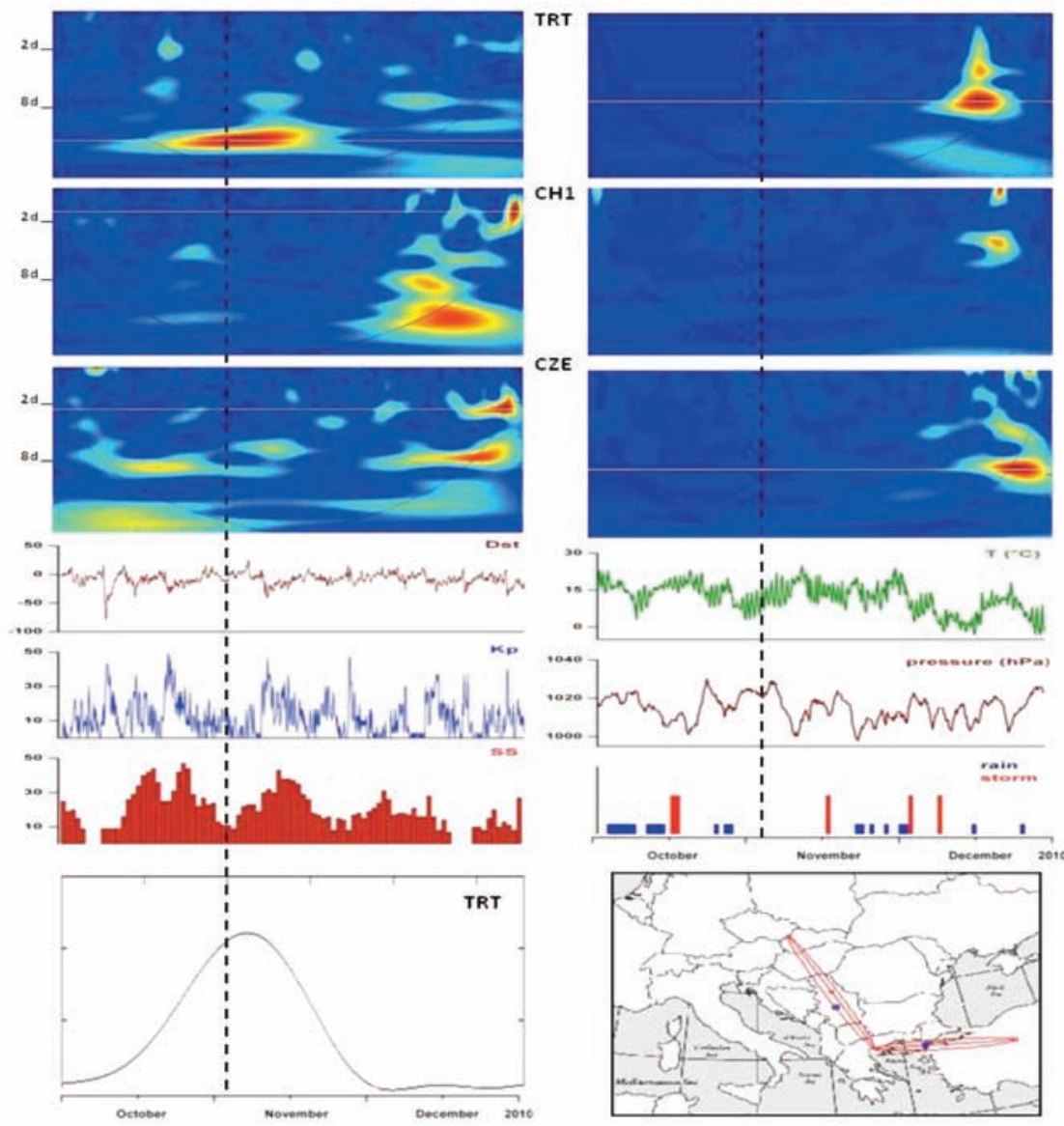

Figure 6. Night-time and day-time spectrograms of the TRT (180 kHz), CH1 $(198 \mathrm{kHz})$ and CZE $(270 \mathrm{kHz})$ transmitter radio signals collected by the GR receiver, together with the geomagnetic/solar and the (local) meteorological data, from October to December 2010. Top panels: Spectrograms of the nighttime (left) and day-time (right) radio signals. Continuous black lines, delimitation of the region (COI) of the wavelet spectra in which edge effects become important [Torrence and Compo 1998]. Centre panels: Dst and Kp geomagnetic indices and solar burst numbers (SS) (left), and air temperature, air pressure and occurrence of rain and storms (right). Bottom panels: Wavelet power corresponding to the Fourier period in which the maximum power was found for the $180 \mathrm{kHz}$ signal (left), and map showing the 5th Fresnel zone of the GR/TRT and GR/CZE paths (right), showing the epicentres of the November 3, 2010, earthquakes that occurred inside these zones. Vertical dashed lines, times of occurrence of these earthquakes. 


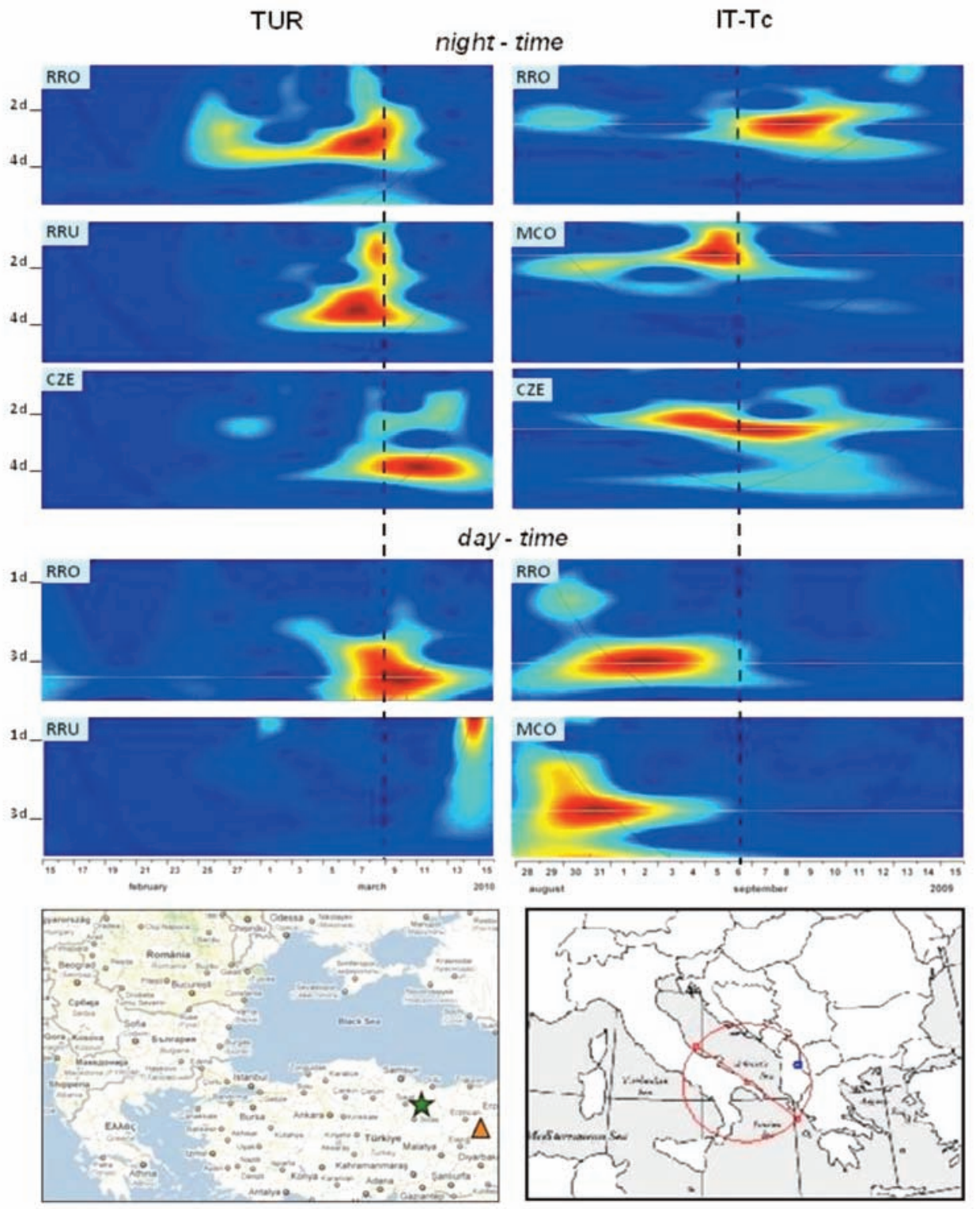

Figure 7. Night-time and day-time spectrograms of some of the radio signals collected by the TUR receiver relative to the $M=6.0$ earthquake that occurred on March 8, 2010 (left), and by the IT-Tc receiver relative to the M = 5.6 earthquake that occurred on September 06, 2009 (right). Top panels: Spectrograms of the night-time data. Centre panels: Spectrograms of the day-time data. Continuous black lines, delimitation of the region (COI) of the wavelet spectra in which edge effects become important [Torrence and Compo 1998]. Bottom panels: Maps showing the TUR receiver (star) and the March 8, 2010, earthquake epicenter (triangle) (left), and the epicenter of the September 06, 2009, earthquake that occurred inside the 300-km radius circle around the IT-Tc receiver (right). Vertical dashed lines, times of occurrence of these earthquakes.

solar and the (local) meteorological data. These two earthquakes happened on the same day (November 3, 2010), and their epicenters were inside the 5th Fresnel zone of the GR/TRT and GR/CZE paths, is indicated. From the spectrograms, three anomalies stand out. The most evident occurs in December. This appears in the different radio signals sampled by the receiver, and it is present both in the night-time and day-time spectrograms. In addition, no similar effects were seen in the data collected by the other receivers of the network. This anomaly appears to be due to the relocation of the GR receiver to a more suitable place, which occurred on December 11, 2010. Another anomaly is present only on the night-time spectrogram of the TRT (180
$\mathrm{kHz}$ ) signal coming from Turkey. The anomaly has a period of about 15 days and it can be clearly related to the earthquake ( $M=5.3)$ that occurred along the GR/TRT path. The one-dimensional plot corresponding to the maximum power of the $180 \mathrm{kHz}$ spectrogram in Figure 6 reveals that the anomaly started 10 days before the earthquake occurrence. Although weak, the last anomaly appears only in the night-time spectrogram of the CZE $(270 \mathrm{kHz})$ signal coming from the Czech Republic. This anomaly is characterized by a period of about 10 days, and it might be related to the earthquake $(M=5.4)$ that occurred along the GR/CZE path. The effects seemed to start 15 days before the earthquake. 


\subsection{Receiver circles}

Figure 7 relates to two cases reported in Table 2, and shows the night-time and day-time spectrograms of some of the radio signals sampled by the TUR and IT-Tc receivers. In both of these cases, clear anomalies with periods of 2-5 days appear in the spectrograms. From the geomagnetic/solar and meteorological data (for simplicity, not shown in Figure 7), no particular activity appears in the anomalous periods. On the contrary, it appears realistic that these anomalies are related to the selected earthquakes. A pre-seismic phase with a duration of a number of days stands out in some of the signals.

\subsection{Transmitter circles}

Finally, in terms of the 'transmitter circles', Figure 8 shows the night-time and day-time spectrograms of the CZE transmitter radio signal (Table 2 ) recorded by the GR, IT-An and IT-Tc receivers, together with the geomagnetic/solar data and meteorological conditions in the transmitter area. Clear anomalies stand out at practically the same time in the night-time and day-time spectrograms. In addition, these do not appear in the spectrograms of the other radio signals recorded by these receivers over the same period. Therefore, these anomalies are clearly related to some local effects. The

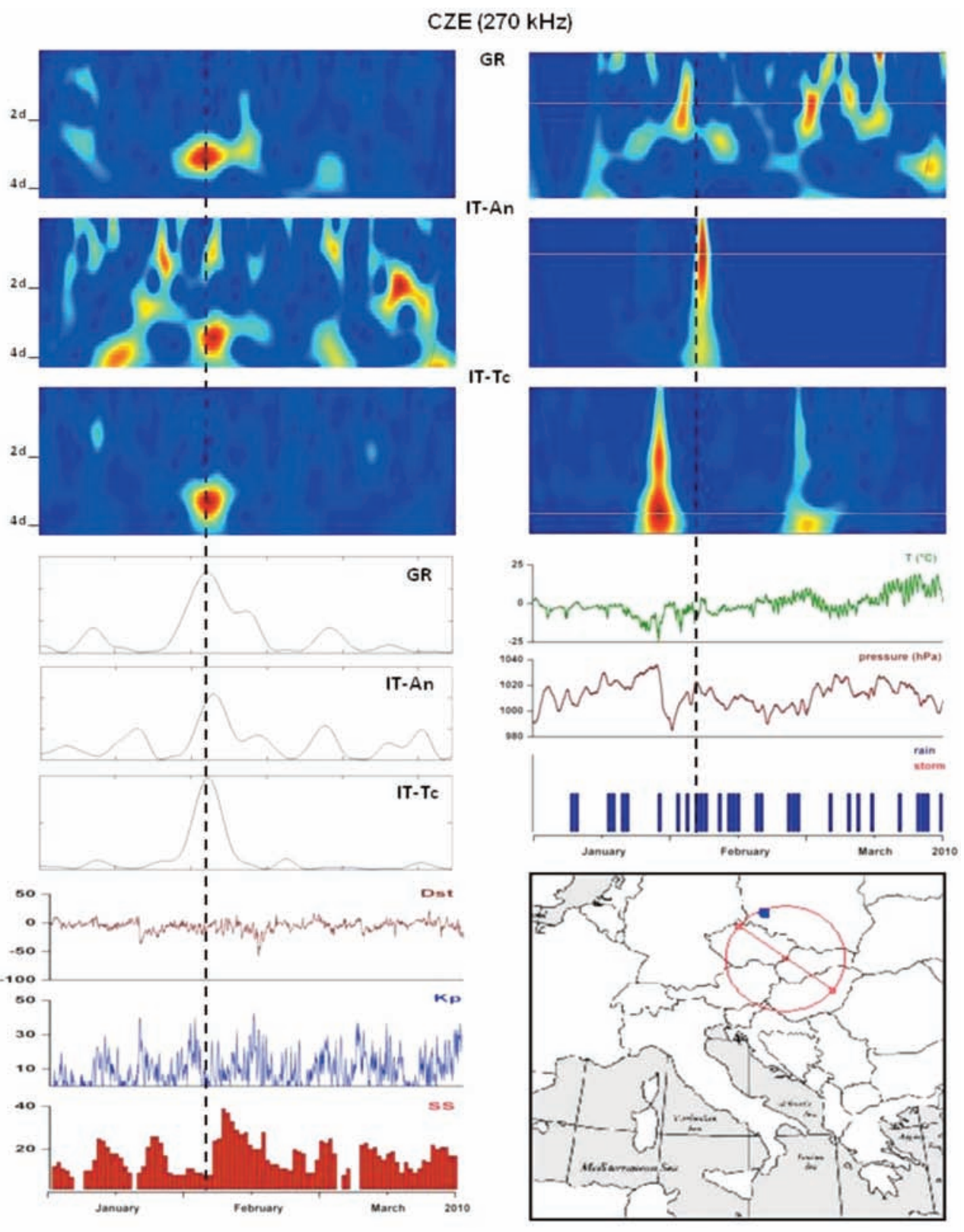

Figure 8. Night-time and day-time spectrograms of the CZE $(270 \mathrm{kHz})$ transmitter radio signals collected by the GR, IT-An and IT-Tc receivers, together with the geomagnetic/solar and the (local) meteorological data, from January to March 2010. Top panels: Spectrograms of the night-time (left) and daytime (right) radio signals. Continuous black lines, delimitation of the region (COI) of the wavelet spectra in which edge effects become important [Torrence and Compo 1998]. Centre left panels: Wavelet powers corresponding to the Fourier period in which the maximum power was found. Bottom left panels: Dst and Kp geomagnetic indices and solar burst numbers (SS). Centre right panels: Air temperature, air pressure and occurrence of rain and storms in the transmitter area. Bottom right panels: Map showing the epicentre of the $M=5.0$ earthquake that occurred on February 6,2010 , inside the 300-km radius circle around the CZE transmitter. Vertical dashed lines, time of occurrence of this earthquake. 
local meteorological conditions in this period are characterized by consistent cooling (down to $-20^{\circ} \mathrm{C}$ ), and this might be the cause of the radio anomalies. At the same time, in this period, an earthquake with $M=5.0$ occurred near to the CZE transmitter, as indicated in Table 2 and shown on the map in Figure 8. The one-dimension wavelet power plots at night-time that are shown in Figure 8 reveal very good correspondence of the anomalies with the occurrence of the earthquake. Therefore, the seismic justification of these anomalies also appears to be consistent. At the moment, we have not found more arguments for accepting one or the other possibility.

\section{Conclusions}

The wavelet analysis of the LF data collected by the European Radio Network has revealed some possible anomalies related to earthquakes. The concurrent analysis of the day-time and night-time signals was useful for the identification of local effects. Except for some doubt in one case, success appears to be obtained in all of the cases related to the $300 \mathrm{~km}$ circles, for both the ground wave and the sky wave, which reveals that this method of analysis has a good sensitivity for the local disturbances produced by the earthquakes. This is consistent with the occurrence of local perturbations in the so called 'preparation-zone'. For the Fresnel cases, two were successful and one failed in the analysis of the sky wave. In the failed case, no correspondence with the geomagnetic/solar or the meteorological situation was seen; thus, general disturbances in the ionosphere should be claimed here. This situation was revealed in summer and it probably indicates that the sensitivity of the wavelet analysis for studying seismic effects in Fresnel zones is low in the summer. This might be a drawback of the method.

The wavelet is only one of the possible methods of analysis for the discovery of anomalies in a dataset. To confirm or to increase the present results, this study will be continued with further investigation of the cases here using other methods, such as residual $\mathrm{dA} / \mathrm{dP}$ analysis [Rozhnoi et al. 2004], principal component analysis [Joliffe 2002] and detrended fluctuation analysis [Peng et al. 1994].

\section{References}

Alperovitch, L. (1997). Perturbation of atmospheric conductivity as a cause of the seismo-ionospheric interaction, Ann. Geophys., 15 (Suppl. 1), Abstract - XXII EGS Gen. Assembly, Vienna, Austria.

Biagi, P. ., R. Piccolo, A. Ermini, S. Martellucci, C. Bellecci, M. Hayakawa, V. Capozzi and S.P. Kingsley (2001a). Possible earthquake precursors revealed by LF radio signals, Nat. Hazard Earth Sys., 1, 99-104.

Biagi, P.F., R. Piccolo, A. Ermini, S. Martellucci, C. Bellecci,
M. Hayakawa and S.P. Kingsley (2001b). Disturbances in LF radiosignals as seismic precursors, Annals of Geophysics, 44 (5/6), 1011-1019.

Biagi, P.F. and M. Hayakawa (2002). Possible premonitory behaviour of LF radiowaves on the occasion of the Slovenia earthquakes ( $M=5.2-6.0-5.1)$ that occurred on March-May 1998, In: Seismo Electromagnetics: Lithosphere-Atmosphere-Ionosphere Coupling, edited by M. Hayakawa and O. Molchanov, Terra Sci. Pub. Co., Tokyo, 249-253.

Biagi, P.F., L. Castellana, T. Maggipinto, R. Piccolo, A. Minafra, A. Ermini, S. Martellucci, C. Bellecci, G. Perna, V. Capozzi, O.A. Molchanov and M. Hayakawa (2005). A possible preseismic anomaly in the ground wave of a radio broadcasting $(216 \mathrm{kHz})$ during July-August 1998 (Italy), Nat. Hazard Earth Sys., 5, 727-732.

Biagi, P.F., L. Castellana, T. Maggipinto, R. Piccolo, A. Minafra, A. Ermini, S. Martellucci, C. Bellecci, G. Perna, V. Capozzi, O.A. Molchanov and M. Hayakawa (2006a). LF radio anomalies revealed in Italy by the Wavelet analysis: Possible preseismic effects during 1997-1998, Phys. Chem. Earth, 31, 403-408.

Biagi, P.F., L. Castellana, T. Maggipinto, A. Ermini, G. Perna and V. Capozzi (2006b). Electric field strength analysis of 216 and $270 \mathrm{kHz}$ broadcast signals recorded during 9 years, Radio Sci., 41, RS4013.

Biagi, P.F., T. Maggipinto, F. Righetti, D. Loiacono, L. Schiavulli, T. Ligonzo, A. Ermini, I.A., Moldovan, A.S., Moldovan, A. Buyuksarac, H.G. Silva, M. Bezzeghoud and M.E. Contadakis (2011). The European VLF/LF radio network to search for earthquake precursors: setting up and natural/man-made disturbances, Nat. Hazards Earth Sys., 11, 333-341.

Daubechies, I. (1992). Ten lectures on wavelets, CBMS NSF Regional Conferences Series in Applied Mathematics SIAM, Philadelphia PA, 61.

Dobrovolsky, I.P., S.I. Zubkov and V.I. Miachkin (1979). Estimation of the size of earthquake preparation zone, Pure Applied. Geophys., 117, 1025-1044.

Hayakawa, M. and H. Sato (1994). Ionospheric perturbations associated with earthquakes, as detected by subionospheric VLF propagation, In: Electromagnetic Phenomena Related to Earthquake Prediction, edited by M. Hayakawa and Y. Fujinawa, Terra Sci. Pub. Co., Tokyo, 391-397.

Hayakawa, M., O.A. Molchanov, T. Ondoh and E. Kawai (1996). The precursory signature effect of the Kobe earthquake on subionospheric VLF signals, J. Comm. Res. Lab., 43, 169-180.

Hayakawa, M., K. Ohta, S. Maekawa, T. Yamauchi, Y. Ida, T. Gotoh, N. Yonaiguchi, H. Sasaki and T. Nakamura (2006). Electromagnetic precursors to the 2004 Mid Niigata Prefecture earthquake, Phys. Chem. Earth, 31, 356- 
364.

Joliffe, I.T. (2002). Principal Component Analysis, New York, USA.

King, Chi-Yu (Ed.) (1984/85): Earthquake hydrology and chemistry, Special Issue, Pure Appl. Geophys., 122, 141618.

Kingsley, S.P., P.F. Biagi, R. Piccolo, V. Capozzi, A. Ermini, Y.M. Khatkevich and E.I. Gordeev (2001). Hydrogeochemical precursors of strong earthquakes: a realistic possibility in Kamchatka, Phys. Chem. Earth, 26, 769774.

Knight, P. (1973). MF propagation: a wave-hop method for ionospheric field strength prediction, Rep.13, 1-20, BBC Research Department, London.

Molchanov, O.A. and M. Hayakawa (1998). Subionospheric VLF signal perturbations possibly related to earthquakes, J.Geophys. Res., 103, 17489-17504.

Molchanov, O.A., A. Rozhnoi, M. Solovieva, O. Akentieva, J.J. Berthelier, M. Parrot, F. Lefeuvre, P.F. Biagi, L. Castellana and M. Hayakawa (2006). Global diagnostic of the ionospheric perturbations related to the seismic activity using the VLF radio-signals collected on the DEMETER satellite, Nat. Hazards Earth Sys., 6, 745-753.

Morgounov, V.A., T. Ondoh and S. Nagai (1994). Anomalous variation of VLF signals associated with strong earthquakes $(\mathrm{M} \geq 7.0)$, In: Electromagnetic Phenomena Related to Earthquake Prediction, edited by M. Hayakawa and Y. Fujinawa, Terra Sci. Pub. Co., Tokyo, 409428.

Peng, C.K., S.V. Buldyrev, S. Havlin, M. Simons, H.E. Stanley and A.L. Goldberger (1994). Mosaic organization of DNA nucleotides, Phys. Rev. E, 49, 1685-1689.

Pulinets, S.A., V.V. Hegai, K.A. Boyarchuk and V.A. Alekseev (1998). The new conception of earthquake prediction, Ann. Geophys., 16 (Suppl 1), Abstract - XXIII EGS Gen. Assembly, Nice, France.

Rikitake, T. (1975). Earthquake precursors, Bull. Seism. Soc. Am., 65, 1133-1162.

Rikitake, T. (1987). Earthquake precursors in Japan: precursor time and detectability, Tectonophysics, 136, 265282.

Roeloffs, E.A. (1988). Hydrologic precursors to earthquakes: A review, Pure App. Geophys., 126, 177-209.

Rotehram, S. (1981a). Ground wave propagation, Part 1: Theory for short distances, IEE Proc. F, Communication, Radar and Signal Process, 128 (5), 275-284.

Rotehram, S. (1981b). Ground wave propagation, Part 2:Theory for medium and long distances and reference propagation curves, IEE Proc. F, Communication, Radar and Signal Process, 128 (5), 285-295, .

Rozhnoi, A., M.S. Solovieva, O.A. Molchanov and M. Hayakawa (2004). Middle latitude $\mathrm{LF}(40 \mathrm{kHz})$ phase variations associated with earthquakes for quiet and disturbed geomagnetic conditions, Phys. Chem. Earth, 29, 589-598.

Rozhnoi, A., O. Molchanov, M. Solovieva, V. Gladyshev, O. Akentieva, J.J. Berthelier, M. Parrot, F. Lefeuvre, M. Hayakawa, L. Castellana and P.F. Biagi (2007). Possible seismo-ionosphere perturbations revealed by VLF signals collected on ground and on a satellite, Nat. Hazard Earth Sys., 7, 617-624.

Strang, G. and T. Nguye (1996). Wavelets and filter banks, Wellesley Cambridge Press, 490 pp.

Torrence, C. and G.P. Compo (1998). A practical Guide to Wavelet Analysis, B. Am. Meteor. Soc., 79 (1), 61-78.

Wyss, M. and R. Dmowska (Eds.)(1997). Earthquake Prediction - State of the Art, Pure Appl. Geophys., 149, 272 pp.

\footnotetext{
* Corresponding author: Flavia Righetti, Università di Bari, Dipartimento di Fisica, Bari, Italy; email: rete.fisica@uniba.it.
}

(C) 2012 by the Istituto Nazionale di Geofisica e Vulcanologia. All rights reserved. 